\title{
IL-6 Quotient (The Ratio of Cerebrospinal Fluid IL-6 to Serum IL-6) as a Biomarker of an Unruptured Intracranial Aneurysm
}

\author{
Joanna Kamińska (iD) \\ Violetta Dymicka-Piekarska (D) \\ Robert Chrzanowski (iD) ${ }^{2}$ \\ Karol Sawicki (iD) ${ }^{2}$ \\ Anna J Milewska (iD ${ }^{3}$ \\ Justyna Zińczuk \\ Marzena Tylicka (iD ${ }^{4}$ \\ Marek Jadeszko ${ }^{2}$ \\ Zenon Mariak iD ${ }^{2}$ \\ Ewa M Kratz iD ${ }^{5}$ \\ Joanna Matowicka-Karna (iD) \\ Johannes Kornhuber (iD ${ }^{6}$ \\ Piotr Lewczuk (iD) ${ }^{6,7}$ \\ Olga M Koper-Lenkiewicz (iD)
}

'Department of Clinical Laboratory Diagnostics, Medical University of Białystok, Białystok, 15-269, Poland; ${ }^{2}$ Department of Neurosurgery, Clinical Hospital of the Medical University of Białystok, Białystok, 15-276, Poland; ${ }^{3}$ Department of Statistics and Medical Informatics, Medical University of Białystok, Białystok, 15-295, Poland; ${ }^{4}$ Department of Biophysics, Medical University of Białystok Białystok, 15-089, Poland; ${ }^{5}$ Department of Laboratory Diagnostics, Division of Laboratory Diagnostics, Faculty of Pharmacy, Wrocław Medical University, Wrocław, 50-556, Poland; ${ }^{6}$ Department of Psychiatry and Psychotherapy, Friedrich-Alexander-Universität ErlangenNürnberg, Universitätsklinikum Erlangen, Erlangen, 91054, Germany; ${ }^{7}$ Department of Neurodegeneration Diagnostics, Medical University of Białystok, Białystok, 15-269, Poland

Correspondence: Joanna Kamińska; Olga M Koper-Lenkiewicz

Department of Clinical Laboratory Diagnostics, Medical University of Bialystok, Waszyngtona I5A St., Białystok, 15-269, Poland

Tel/Fax +48 857468584

Email joanna.kaminska@umb.edu.pl; o.koper@wp.pl
Background: Studies conducted so far have focused mainly on the assessment of IL-6 levels in patients with ruptured brain aneurysms. Carrying out detailed studies in patients with un-ruptured brain aneurysms (UIA) would be extremely important, as it would answer the question of whether IL-6 plays also a role in primary aneurysm formation and growth.

Methods: IL-6, S100, NSE, and albumin concentrations in 67 UIA patients and 17 individuals without vascular lesions in the brain were tested using in vitro diagnostic immunoassays according to the manufacturers' instructions. IL-6 Quotient was calculated by dividing cerebrospinal fluid (CSF) IL-6 by serum IL-6.

Results: We showed that IL-6 Quotient was significantly higher in UIA patients (1.78) compared to the control group $(0.87 ; \mathrm{p}<0.001)$. Multivariate logistic regression analysis demonstrated that a growth in IL-6 Quotient increases the probability of UIA diagnosis. In UIA patients CSF IL-6 concentration was significantly higher $(4.55 \mathrm{pg} / \mathrm{ml})$ compared to the serum concentration $(2.39 \mathrm{pg} / \mathrm{ml} ; \mathrm{p}<0.001)$. In both the study and control group, the bloodbrain barrier was intact, thus the CSF-blood gradient of the IL-6 concentration in UIA patients was likely to be the expression of local synthesis of the cytokine within the central nervous system. Patients with multiple brain aneurysms had significantly higher CSF IL-6 levels $(5.08 \mathrm{pg} / \mathrm{ml})$ compared to individuals with a single aneurysm $(4.14 \mathrm{pg} / \mathrm{ml} ; \mathrm{p}=0.0227)$. Conclusion: This totality of the may suggest IL-6 as a biomarker for UIA formation; however, further studies are needed to unequivocally confirm clinical application of IL-6 concentration evaluation.

Keywords: cerebrospinal fluid, CSF, interleukin 6, IL-6, unruptured intracranial aneurysm, UIA, IL-6 Quotient

\section{Background}

An intracranial aneurysm (IA) can lead to a life-threatening subarachnoid hemorrhage (SAH), which is lethal in approximately $50-60 \%$ of cases and significantly disabling for those who survive. ${ }^{1}$ Recognition of the factors involved in the pathomechanism of IA formation and growth would be extremely important, as it could indicate a direction for preventive treatment of IA before its rupture. ${ }^{2}$

IA formation begins with hemodynamically induced inflammatory dysfunction of the endothelium, which is the cause, not the consequence, of the presence and rupture of the aneurysm. ${ }^{2}$ There is a correlation between an abnormal remodeling of the cerebral vessel and a wall shear stress (WSS) gradient. ${ }^{3}$ Hemodynamical alternations lead to local inflammatory changes in endothelial cells (ECs) and 
vascular smooth muscle cells (VSMCs). ${ }^{2,4-7}$ The formation of an aneurysm begins when WSS initiates the production of the nuclear factor kappa-light-chain-enhancer of activated B cells (NF-kappa B) being a transcription factor that initiates the process of inflammation. WSS also leads to infiltration of inflammatory cells (macrophages, neutrophils, lymphocytes) and activation of matrix metalloproteinases (MMPs) in the aneurysm wall, which in turn leads to the release of inflammatory cytokines, adhesion molecules, chemokines, immunoglobulins, proteases, reactive oxygen species (ROS), and complement components. ${ }^{2,8,9}$ At the same time, mechanisms protecting the arterial wall are activated. The VSMCs' inner membrane hyperplasia is induced, aimed at blurring the defects of the arterial walls and increasing the synthesis of nitric oxide (NO), which provides direct protection of the artery wall and inhibits the activation of NF-kB. ${ }^{8,10}$

Studies indicate that among inflammatory factors, interleukin 6 (IL-6) seems to play a critical role in the formation and progression of IA..$^{3,11-13}$ It is produced eg by monocytes/macrophages and lymphocytes infiltrating the arterial wall. ${ }^{2,14,15}$ Animal model experiments show that IL-6 applied locally is a potent basal artery constrictor. ${ }^{16}$ Human studies suggest that the evaluation of IL-6 concentration in the cerebrospinal fluid (CSF) may help predict vasospasm following aneurysmal SAH. ${ }^{11,17-19}$ Moreover, it has been shown that elevated levels of IL-6 in the blood during the first three days after rupture of the brain aneurysm is an early and independent predictor of poor clinical prognosis within 30 days after aneurysmal $\mathrm{SAH}^{20}$

It is extremely important to identify signaling pathways that are responsible for the development and rupture of an aneurysm. ${ }^{2}$ Studies conducted so far have focused mainly on the assessment of IL-6 levels in patients with ruptured brain aneurysms. Carrying out detailed studies in patients with unruptured brain aneurysms (UIA) would be extremely important, as it would answer the question of whether IL-6 also plays a role in primary aneurysm formation and growth.

Since the disturbance of the integrity of the Bloodbrain barrier (BBB) and the permeability of the Bloodcerebrospinal fluid barrier (BCSFB) may affect the final concentration of IL-6 in the CSF, we assessed the concentration of this cytokine depending on the condition of the above-mentioned barriers in the first step. In the next step, we compared CSF IL-6 concentration to serum values in UIA patients, to check whether IL-6 synthesis occurs locally as a result of changes in the cerebral vessel. In our previous study, we observed that the calculation of IL8 Quotient (concentration of CSF protein divided by serum protein) was a satisfactory approach to distinguish UIA patients from individuals without vascular lesions in the brain. ${ }^{21}$ Finally, we calculated IL-6 Quotient in UIA patients compared to the control group as well as taking into account the size and the number of aneurysms.

\section{Methods}

\section{Subjects}

The study group consisted of 67 patients: 53 females/14 males, with a median age of 59 years. The patients were operated on due to an unruptured brain aneurysm between July 2015 and October 2019 at the Department of Neurosurgery at the Clinical Hospital of the Medical University of Bialystok. All patients underwent craniotomy and direct surgical clipping of unruptured intracranial aneurysm (UIA). In all patients, the aneurysms were asymptomatic and located at the anterior part of the Willis' Circle. The decision for intervention was made only after careful consideration of the risk of aneurysm rupture vs the inherited risk of surgical procedure. The commonly used guidelines (eg, size $>5 \mathrm{~mm}$ and/or irregular shape), representing the increased risk of rupture ${ }^{22}$ and the patients' opinions were taken into consideration when making the decision. The presence of UIA was established with MR angiography in 21 patients, and with CT angiography in 33 patients. Thirteen individuals required confirmation with contrast digital angiography to pinpoint the location, size, and shape of the aneurysm. Table 1 contains the aneurysms' geometrical information in the study group.

Exclusion criteria comprised neurodegenerative conditions like multiple sclerosis, neuroinfection and a brain tumor in the medical history, surgery or major trauma in preceding months, antibiotics, anti-inflammatory, or corticosteroid administration in preceding months.

The comparative group was made up of 17 subjects (7 males/10 females, mean age 66 years, quartiles 57-68 years), suffering from trigeminal neuralgia due to an anatomical conflict between the trigeminal nerve and a cerebellar artery. All patients in this group showed to be unresponsive to conservative treatment and were qualified for posterior fossa craniotomy and microvascular decompression. The procedure consisted of the exposition of the conflict area at the cerebro-pontine angle, disinsertion of the compressing 
Table I Aneurysms' Geometry Information in the Study Group

\begin{tabular}{|l|c|}
\hline \multicolumn{2}{|c|}{ Aneurysm Geometry Characteristics } \\
\hline Aneurysm Location: & No (\%) \\
\hline MCA & $54(81 \%)$ \\
MCA, ICA & I (2\%) \\
ICA & $9(13 \%)$ \\
AcomA & $3(4 \%)$ \\
\hline Side: & $31(46 \%)$ \\
\hline Left & $36(54 \%)$ \\
Right & $n=533^{\#}$ \\
\hline Aneurysm size [mm]: & $5.3 \mathrm{~mm}$ \\
Median & $21(40 \%)$ \\
< $5 \mathrm{~mm}$ & $30(57 \%)$ \\
$5-9.9 \mathrm{~mm}$ & $2(3 \%)$ \\
\hline$\geq$ I0 mm & $38(57 \%)$ \\
\hline Number of aneurysms: & $29(43 \%)$ \\
\hline Single & \\
Multiple &
\end{tabular}

Notes: ${ }^{\#}$ In the case of 14 UIA patients there was no history of the size of the operated aneurysm.

Abbreviations: AcomA, anterior communicating artery; ICA, internal carotid artery; MCA, middle cerebral artery.

artery from the trigeminal nerve, displacement of the artery, and finally its fixation to the cerebellar tentorium with a binding agent (Tachosil ${ }^{\circledR}$, Takeda, Linz, Austria). The exclusion criteria from the control group were consistent with those of the study group. All patients before surgery had fasting basic laboratory tests done between 6:00 AM and 7:00 AM. Table 2 presents the characteristics of the study and control groups. In the analyzed basic laboratory parameters, we observed a significant difference in the results obtained for $\mathrm{WBC}$, MPV, INR, $\mathrm{K}^{+}, \mathrm{CRP}$ in patients with UIA compared to the control group, but the results found in both groups for these parameters are within the reference range, indicating no clinical significance for the observed differences.

\section{Sample Collection and Storage}

Surgical procedures were conducted in the usual manner: under general anesthesia with the patient's head immobilized in a three-pin Mayfield head holder. Skin incision preceded lifting of bone flap and lancing of dura mater, allowing for visualization of the arachnoid membrane and subarachnoid space. Subarachnoid space was carefully opened with the aid of an operating microscope, and inflowing CSF removed with a single- use, sterile syringe, and soft venous catheter. Particular care was taken to prevent any contamination of the CSF with blood or warm saline solution used as irrigation. All the aforementioned steps were taken at the start of each procedure, before any bleeding could occur. In all CSF samples, the red blood cell count (RBC) was measured using UF-4000 (Sysmex, Kobe, Japan). Only CSF samples with $\mathrm{RBC}$ count that equal $0 / \mu \mathrm{L}$ were used for further analysis.

Test tubes without anticoagulant (S-Monovette, SARSTEDT) were used in patients' blood samples $(2.7 \mathrm{~mL})$ collection. Blood was centrifuged for 20 minutes at $1000 \mathrm{x} g$ to obtain serum within 30 minutes after the venipuncture. Also, CSF samples were centrifuged for 20 minutes at $1000 \mathrm{x}$ g. Obtained serum and CSF supernatant were aliquoted and placed in storage at $-75^{\circ} \mathrm{C}$ before use. Before the assessment, all specimens were gradually defrosted and mixed using a Vortex. The concentration of IL-6 in CSF and serum was assessed for 61 patients and the concentration of S100 for 53 patients due to a sufficient quantity of samples not being available for analysis. The remaining analysis was performed for all $(n=67)$ patients included in the research.

\section{CSF and Serum IL-6 Concentration Evaluation}

The concentration of IL-6 in serum and CSF was tested using in vitro diagnostics electrochemiluminescence immunoassay (ECLIA) on the COBAS e411 analyzer (Roche Diagnostics, Penzberg, Germany) according to the manufacturer's instructions. Serum and CSF samples were not diluted before analysis. The detection range of the assay test is $1.5-5000 \mathrm{pg} / \mathrm{ml}$. The cytokines: IL- $1 \alpha$, $-1 \beta,-2,-3,-4,-8$, interferon- $\gamma$, and tumor necrosis factor- $\alpha$ in the concentration of $50 \mathrm{ng} / \mathrm{ml}$ do not show cross-reactions. The assay is unaffected by icterus (bilirubin $<680 \mu \mathrm{mol} / \mathrm{l}$ or $<40 \mathrm{mg} / \mathrm{dl}$ ), hemolysis (hemoglobin $<0.621 \mathrm{mmol} / \mathrm{l}$ or $<1.0 \mathrm{~g} / \mathrm{dl}$ ), lipemia (lipid $<1500 \mathrm{mg} / \mathrm{dl}$ ), and biotin $(<123 \mathrm{nmol} / 1$ or $<30 \mathrm{ng} / \mathrm{ml})$.

\section{CSF Total Protein and CSF and Serum Albumin Evaluation}

The concentration of total protein in CSF and albumin in CSF and serum was tested using an automatic biochemical analyzer ACCENT-200 (PZ Cormay S.A., Motycz, Poland). Total protein concentration in CSF was assessed 
using the pyrogallol red colorimetric method. Albumin concentration in CSF and serum was measured by the immunoturbidimetric method. The tests were performed according to the manufacturer's instructions.
CSF and Serum SI00 Protein (S100) and Neuron-Specific Enolase (NSE) Evaluation

CSF and serum S100 concentration was tested on the COBAS e411 analyzer (Roche Diagnostics, Penzberg,

Table 2 Demographical and Clinical Characteristics of Unruptured Intracranial Aneurysm (UIA) Patients and Control Group

\begin{tabular}{|c|c|c|c|c|c|}
\hline \multicolumn{6}{|c|}{ Clinical Characteristics of the Study Group } \\
\hline & UIA Group $(n=67)$ & Control C & oup $(n=17)$ & \multirow{2}{*}{\multicolumn{2}{|c|}{ p-value }} \\
\hline & \multicolumn{3}{|c|}{ Median (interquartiles) } & & \\
\hline Age [years] & \multicolumn{2}{|c|}{$59(5 I-66)$} & \multicolumn{2}{|c|}{$66(57-68)$} & 0.0550 \\
\hline Gender: Female /Male & \multicolumn{2}{|c|}{$53 / 14$} & \multicolumn{2}{|c|}{$10 / 7$} & $0.1263^{\&}$ \\
\hline Caucasian ethnicity & \multicolumn{2}{|c|}{$67(100)$} & \multicolumn{2}{|c|}{$17(100)$} & 1.0000 \\
\hline Weight $[\mathrm{kg}]$ & \multicolumn{2}{|c|}{$70(63-80)$} & \multicolumn{2}{|c|}{$82(68-87)$} & 0.1045 \\
\hline Growth [m] & \multicolumn{2}{|c|}{$1.64(1.60-1.68)$} & \multicolumn{2}{|c|}{$1.68(1.60-1.72)$} & $0.407 I$ \\
\hline BMI & \multicolumn{2}{|c|}{$25.91(23.31-30.08)$} & \multicolumn{2}{|c|}{$28.83(25.69-32.03)$} & 0.1458 \\
\hline Systolic BP [mmHg] & \multicolumn{2}{|c|}{$137(123-150)$} & \multicolumn{2}{|c|}{$143(132-152)$} & 0.3140 \\
\hline Diastolic BP $[\mathrm{mmHg}]$ & \multicolumn{2}{|c|}{$80(77-90)$} & \multicolumn{2}{|c|}{$80(73-95)$} & 0.8340 \\
\hline Heart rate [minute] & \multicolumn{2}{|c|}{$74(66-8 I)$} & \multicolumn{2}{|c|}{$74(64-8 I)$} & 0.6994 \\
\hline \multicolumn{6}{|c|}{ Basic Laboratory Parameters } \\
\hline WBC $\left[\times 10^{3} / \mu 1\right]$ & \multicolumn{2}{|c|}{$7.33(6.27-8.70)$} & \multicolumn{2}{|c|}{$5.97(4.77-7.52)$} & 0.0110 \\
\hline $\mathbf{R B C}\left[\times 10^{6} / \mu 1\right]$ & \multicolumn{2}{|c|}{$4.45(4.18-4.77)$} & \multicolumn{2}{|c|}{$4.51(4.45-4.60)$} & 0.5218 \\
\hline HGB [g/dl] & \multicolumn{2}{|c|}{ I3.50 (|2.80-|4.20) } & $13.50(12.90$ & & 0.8947 \\
\hline HCT [\%] & 39.40 & $7.60-42.10)$ & 40.20 & & 0.8083 \\
\hline PLT $\left[\times 10^{3} / \mu 1\right]$ & 235 & (95-276) & $263(238$ & & 0.0626 \\
\hline MPV [fl] & 10.8 & (0.3-11.4) & $10.3(9.8$ & & 0.0113 \\
\hline P-LCR[\%] & 31.8 & $27.1-36.7)$ & $28.2(24.1$ & & 0.0522 \\
\hline PT $[\mathrm{s}]$ & 12.3 & II.9-12.8) & I $2.7(12.1$ & & 0.1367 \\
\hline INR & 0.92 & $0.89-0.97)$ & $0.99(0.9)$ & & 0.0469 \\
\hline APTT [s] & 28.2 & 26.9-29.7) & 27.1 & & 0.4727 \\
\hline Fibrinogen $[\mathrm{mg} / \mathrm{dl}]$ & 339 & $295-390)$ & $307(273$ & & 0.1458 \\
\hline $\mathrm{Na}^{+}[\mathrm{mmol} / \mathrm{l}]$ & 138 & $|37-| 40)$ & $139(136$ & & 0.6912 \\
\hline $\mathbf{K}^{+}[\mathrm{mmol} / \mathrm{l}]$ & 4.4 & $(4.1-4.7)$ & $4.7(4.4$ & & 0.0456 \\
\hline Glucose $[\mathrm{mg} / \mathrm{dl}]$ & 96 & 88-107) & $99(89$ & & 0.9648 \\
\hline Urea $[\mathrm{mg} / \mathrm{dl}]$ & & (25-39) & $28(22$ & & 0.7574 \\
\hline Creatinine $[\mathrm{mg} / \mathrm{dl}]$ & 0.76 & $0.67-0.85)$ & $0.80(0.70$ & & 0.6831 \\
\hline $\mathbf{C R P}[\mathrm{mg} / \mathrm{l}]$ & 0.70 & $0.40-1.90)$ & $1.75(1.50$ & & 0.0409 \\
\hline & Factors n (\%) & & & & p-value \\
\hline Smoking tobacco" & & $(52)$ & $5(29$ & & 0.0923 \\
\hline Drinking alcohol@ & & $(12)$ & $3(18$ & & 0.5334 \\
\hline Diabetes & & $(5)$ & $4(2$ & & $0.0407^{\&}$ \\
\hline Obesity $^{\$}$ & & $3(27)$ & $7(4)$ & & 0.2492 \\
\hline Hypertension & & $(69)$ & $8(47$ & & 0.0970 \\
\hline Cardiovascular diseases & & $(15)$ & $0(0$ & & $0.2013^{8}$ \\
\hline Head injury & & (II) & $0(0$ & & $0.3678^{\&}$ \\
\hline
\end{tabular}

Notes: ${ }^{8}$ Yates' chi-square, ${ }^{\#}$ more than 20 cigarettes a day for over 12 months and more, ${ }^{@}$ more often than occasionally, ${ }^{\$}$ body mass index $(\mathrm{BMI})>30.0\left(\mathrm{~kg} / \mathrm{m}^{2}\right)$, the bold font of $p$-value means that it is statistically significant $(p<0.05)$. Conversion factors to $S I$ units are as follows: for WBC -1.0 , for RBC -1.0, for HGB -10.0 , for PLT -1.0 , for glucose -0.0555 , for creatinine -88.4 . The $\mathrm{p}$-value of $<0.05$ is considered statistically significant.

Abbreviations: APTT, activated partial thromboplastin; eGFR, estimated glomerular filtration rate; HCT, hematocrit; HGB, hemoglobin; INR, International Normalized Ratio; Time; $\mathrm{K}^{+}$, potassium ion; MPV, mean platelet volume; $\mathrm{Na}^{+}$, sodium ion; P-LCR, platelet-large cell ratio; PLT, platelet count, PT, prothrombin time; RBC, red blood cell count; SAH, subarachnoid hemorrhage; UIA, unruptured intracranial aneurysm; WBC, white blood cell count. 
Germany) according to the manufacturer's instructions. The assay is dedicated to the quantitative determination of S100 (S100 A1B and S100 BB) in human samples. Detection range is between 0.005 and $39 \mu \mathrm{g} / \mathrm{l}$. The test is unaffected by icterus (bilirubin $<680 \mu \mathrm{mol} / 1$ or $<40 \mathrm{mg} / \mathrm{dl}$ ), hemolysis (hemoglobin $<0.621 \mathrm{mmol} / 1$ or $<1.0 \mathrm{~g} / \mathrm{dl}$ ), lipemia (lipid $<1500 \mathrm{mg} / \mathrm{dl}$ ), and biotin ( $<205 \mathrm{nmol} / 1$ or $<50 \mathrm{ng} / \mathrm{ml}$ ).

CSF and serum NSE concentrations were tested on the COBAS e411 analyzer (Roche Diagnostics, Penzberg, Germany) according to the manufacturer's instructions. Detection range is between 0.050 and $370 \mathrm{ng} / \mathrm{ml}$. The cytokines: IL- $1 \alpha,-1 \beta,-2,-3,-4,-8$, interferon- $\gamma$, and tumor necrosis factor- $\alpha$ in the concentration of $50 \mathrm{ng} / \mathrm{ml}$ do not show cross-reactions. The assay is unaffected by icterus (bilirubin $<1231 \mu \mathrm{mol} / \mathrm{l}$ or $<72 \mathrm{mg} / \mathrm{dl}$ ), hemolysis (hemoglobin $<0.621$ $\mathrm{mmol} / \mathrm{l}$ or $<1.0 \mathrm{~g} / \mathrm{dl}$ ), lipemia (triglycerides $<22.8 \mathrm{mmol} / \mathrm{l}$ or $<2000 \mathrm{mg} / \mathrm{dl}$ ), and biotin ( $<409 \mathrm{nmol} / \mathrm{l}$ or $<100 \mathrm{ng} / \mathrm{ml})$.

\section{Statistical Analysis}

The obtained results were analyzed with the use of the STATISTICA 12.0 PL software (StatSoft Inc., Tulsa, USA) and GraphPad Prism 5.0 (GraphPad Software, San Diego, USA). They did not follow the normal distribution $\left(\mathrm{X}^{2}\right.$-test $)$, thus nonparametric statistical analyses were applied. The $\mathrm{X}^{2}$ test with Yates correction was used to investigate whether patients were gender-matched and whether there were any differences regarding risk factors. The Mann-Whitney test was used to compare two independent samples. Correlation coefficients were obtained by applying Spearman's rank correlation. Values for continuous variables are presented as median with quartiles. A receiver operator characteristic (ROC) curve was generated to determine the performance of IL-6 level evaluation in order to distinguish between the study and control group. The Youden index, a function of sensitivity and specificity, indicated an optimal trade-off between these two (cut-off point) for the parameters tested. Logistic regression analysis was performed to find out if IL-6 may be predictive in UIA diagnosis. Differences were considered significant for $\mathrm{p}<0.05$.

\section{Results}

\section{Blood-Cerebrospinal Fluid Barrier (BCSFB) Permeability Does Not Influence CSF IL-6 Concentration and IL-6 Quotient in UIA Patients}

Proper BCSFB permeability is related to the CSF flow rate. Albumin Quotient (QAlb), which is equal to albumin concentration in the CSF divided by serum albumin concentration, reflects changes in the CSF flow rate. ${ }^{23,24}$ We found that median QAlb was slightly lower in UIA patients (5.77 $\left.\mathrm{x} 10^{-3}\right)$ compared to the control group $\left(6.23 \times 10^{-3}\right)$, but the difference was insignificant (Figure 1), indicating that these two groups did not differ with regard to BCSFB permeability.

Considering that QAlb is age-dependent, ${ }^{25}$ we calculated the upper limit of QAlb for each patient individually (for detailed information on how to calculate the upper limit of QAlb please refer to ${ }^{21}$ ). Based on the QAlb value we divided UIA patients into those with proper BCSFB permeability $(n=51)$ and those with QAlb above the upper limit $(n=16)$. We found that neither CSF IL-6 concentration nor IL-6 Quotient differs between patients with QAlb $<$ the upper limit compared to patients with QAlb $>$ the upper limit (Figure 2A and B). Also, Spearman's rank correlation coefficient analysis did not reveal any relationship between QAlb and CSF IL-6 or IL-6 Quotient ( $\mathrm{p}>0.05$ ).

\section{UIA Patients Have Proper Blood-Brain Barrier (BBB) Integrity and This Barrier Does Not Influence IL-6 Results}

Well-established markers of BBB integrity are the brainrelated proteins $\mathrm{S} 100 \mathrm{~B}$ and NSE, which are released into peripheral circulation in the case of a damaged barrier. ${ }^{26,27}$ We found that UIA patients had both significantly lower

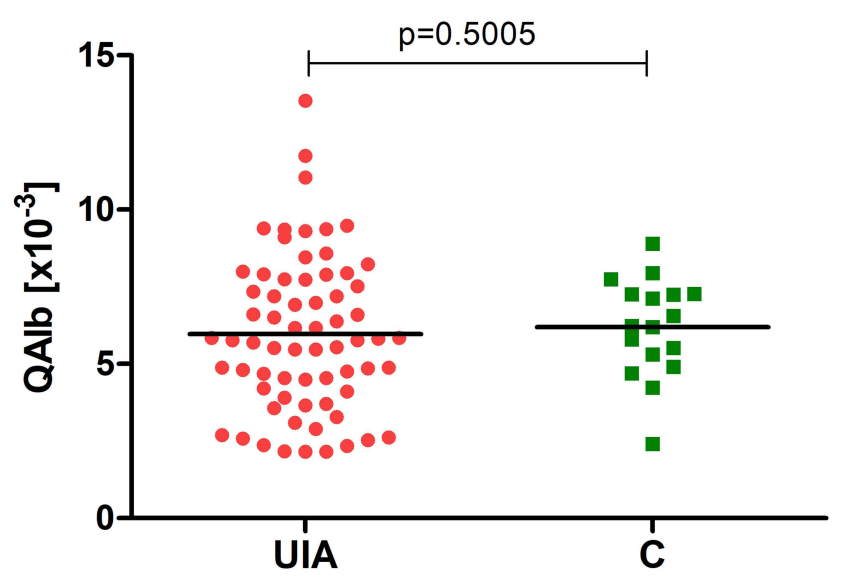

Figure I Albumin quotient (QAlb) in UIA patients compared to the control group. Notes: QAlb - Albumin Quotient calculated by dividing albumin concentration in the cerebrospinal fluid (CSF) by the albumin concentration in the serum. Due to the fact that albumin concentration in the serum $[\mathrm{g} / \mathrm{dl}]$ is much higher than in the CSF $[\mathrm{mg} / \mathrm{dl}]$, it is expressed in different units. Dividing different units by one another is a mistake, thus when calculating the QAlb, units should be unified. This division results in a fraction factor which is inconvenient in everyday practice, ${ }^{24}$ therefore QAlb is quoted as an integer multiplied by $10^{-3}$.

Abbreviations: UIA, unruptured intracranial aneurysm; C, control group; $\mathrm{p}$, P-value. 

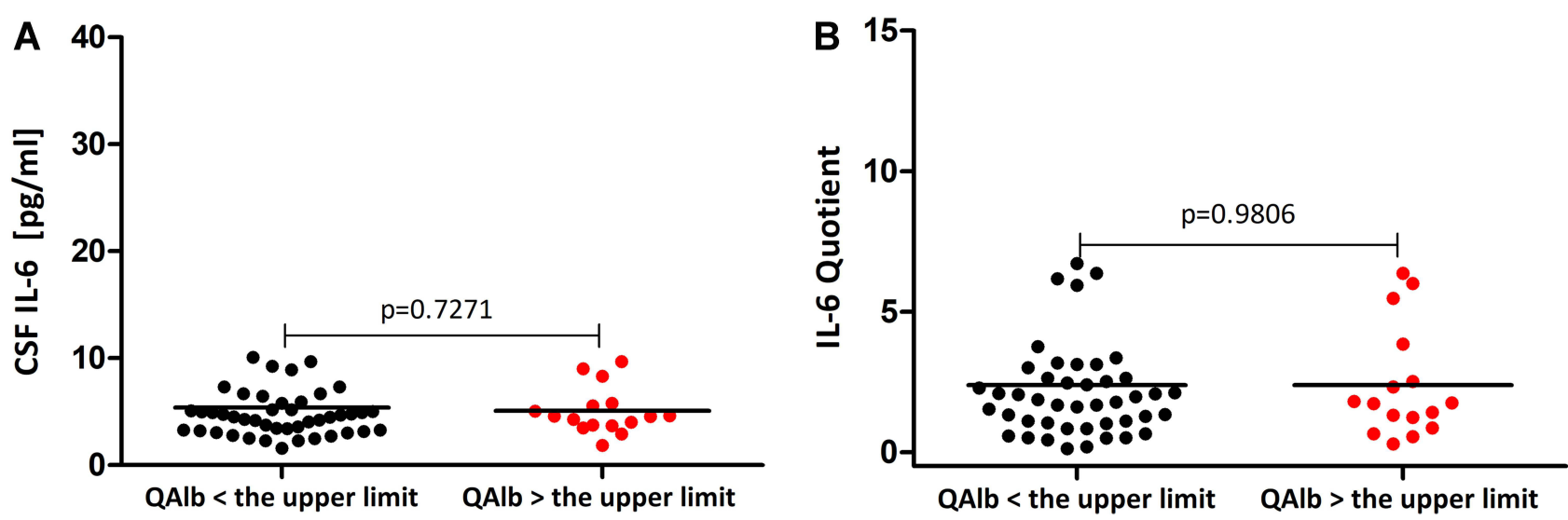

Figure 2 (A) CSF IL-6 concentration depending on QAlb in UIA patients. (B) IL-6 Quotient depending on QAlb in UIA patients.

Notes: QAlb depends on age; for patients between 15 and 60 years old it is calculated using the following formula: QAlb $=[($ age in years $/ 15)+4] \times 10^{-3}$; the upper limit of QAlb is $<8.00 \times 10^{-3}$ for patients $>60$ years of age; ${ }^{24}$.

Abbreviations: CSF, cerebrospinal fluid; IL-6, interleukin 6; IL-6 Quotient, CSF IL-6 concentration divided by serum IL-6 concentration; UIA, unruptured intracranial aneurysm; QAlb, Albumin Quotient calculated by dividing CSF albumin concentration by serum albumin concentration; $p$, $p$-value.
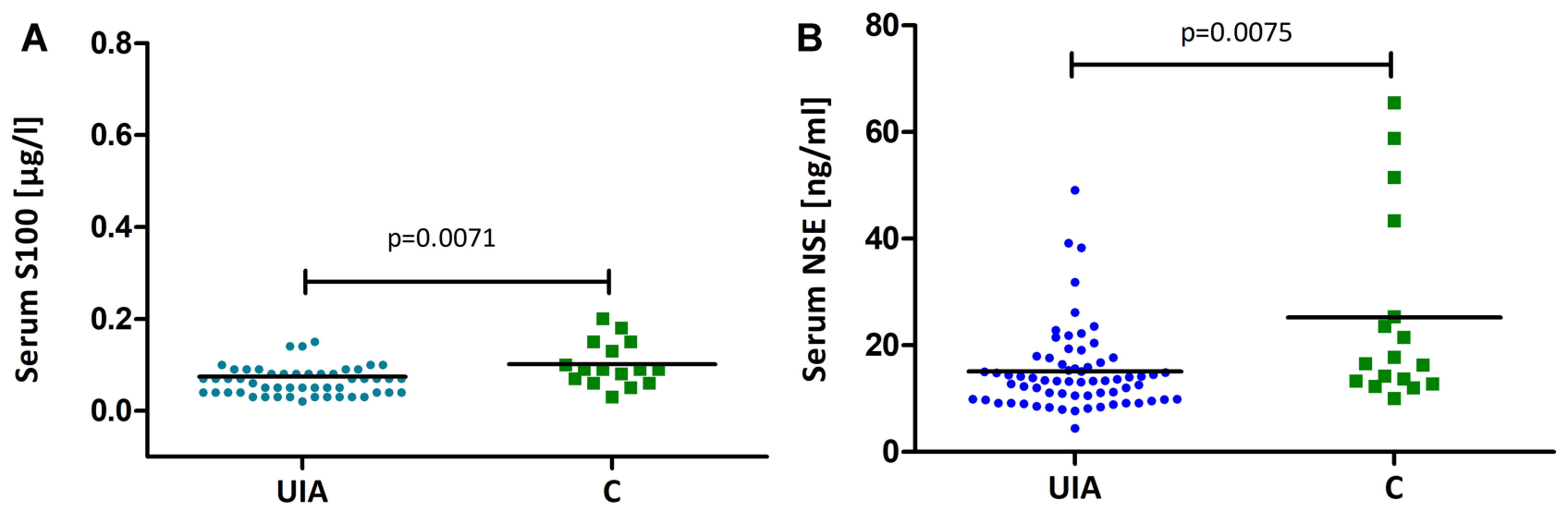

Figure 3 (A) Serum SI00 concentration in UIA patients compared to the control group. (B) Serum NSE concentration in UIA patients compared to the control group. Abbreviations: SI00, SI00 protein; NSE, neuron-specific enolase; UIA, unruptured intracranial aneurysm; C, control group; $\mathrm{p}$, P-value.

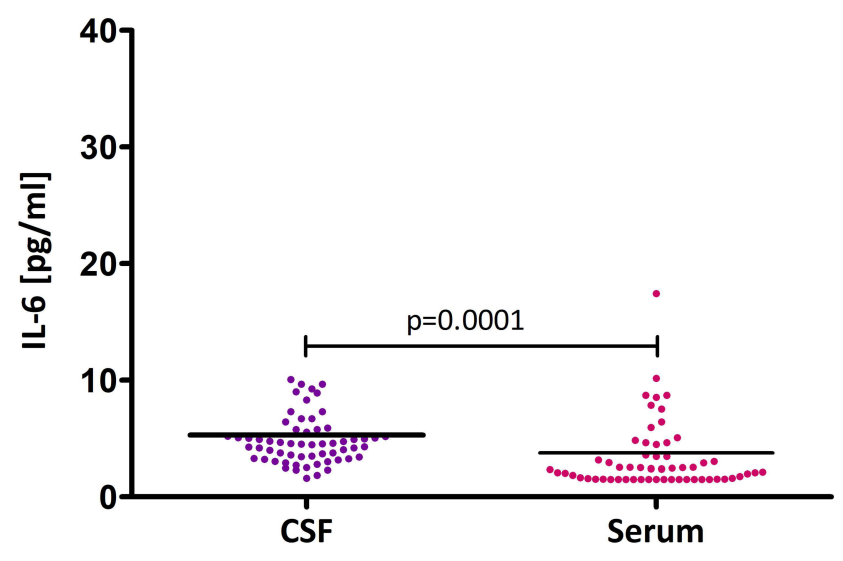

Figure 4 CSF IL-6 concentration compared to serum IL-6 concentration in UIA patients.

Abbreviations: CSF, cerebrospinal fluid; IL-6, interleukin 6; UIA, unruptured intracranial aneurysm; $p$, p-value.

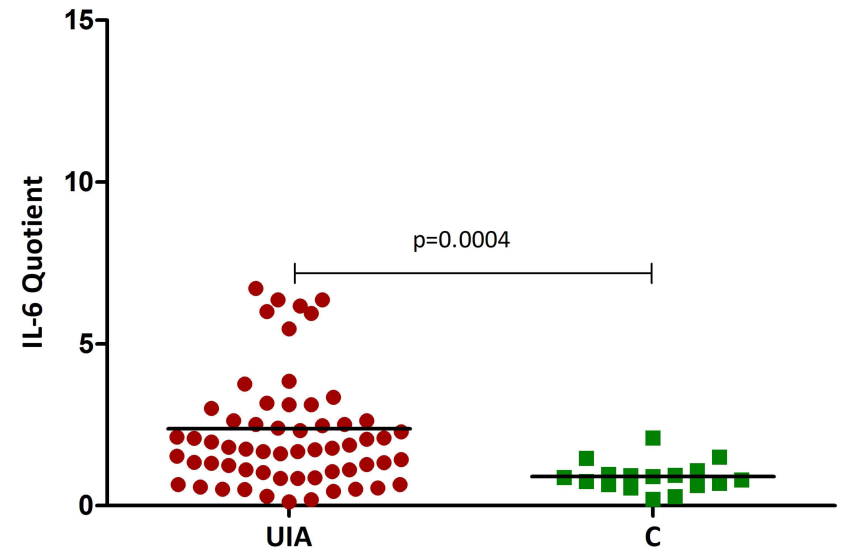

Figure 5 IL-6 Quotient in UIA patients compared to the control group. Abbreviations: IL-6, interleukin 6; IL-6 Quotient, cerebrospinal fluid IL-6 concentration divided by serum IL-6 concentration; UIA, unruptured intracranial aneurysm; C, control group; p, p-value. 


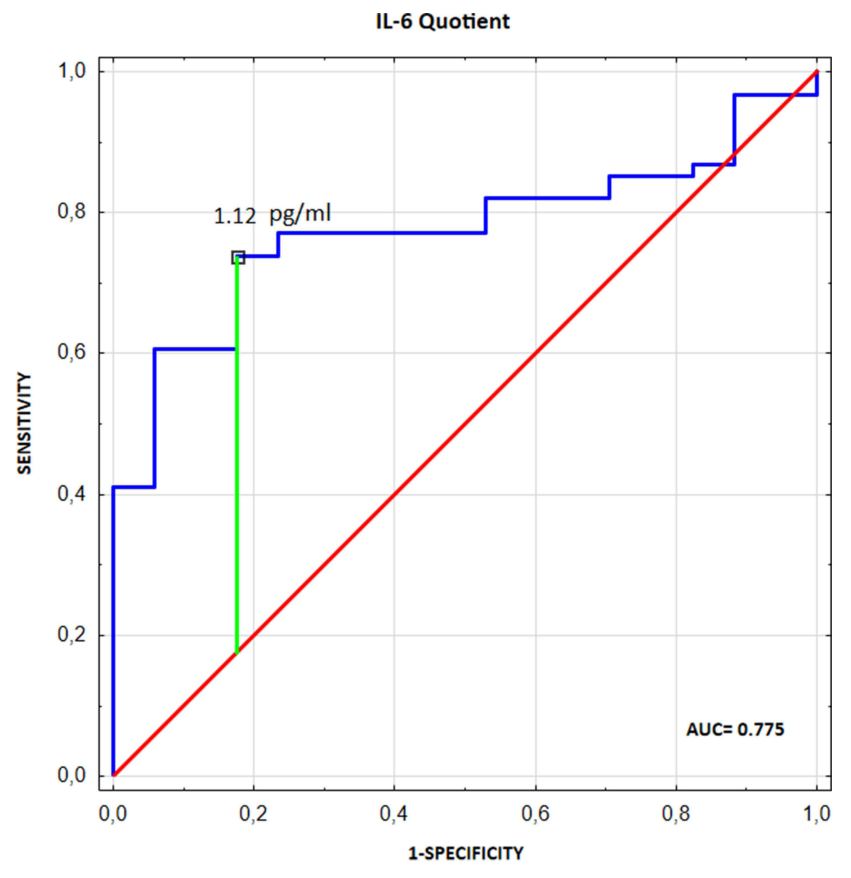

Figure 6 The diagnostic utility analysis of IL-6 Quotient evaluation in differentiating UIA patients from individuals without vascular lesions in the brain.

Notes: ROC curve for IL-6 Quotient $(A U C \pm S E=0.775 \pm 0.053, p<0.0001$; $Y I=0.56$; $\mathrm{SE}=74 \% ; \mathrm{SP}=82 \% ; \mathrm{AC}=76 \% ; \mathrm{PPV}=94 \% ; \mathrm{NPV}=47 \%$ ) for differentiating UIA patients from individuals without vascular lesions in the brain.

Abbreviations: IL-6 Quotient, cerebrospinal fluid IL-6 concentration divided by serum IL-6 concentration; UIA, unruptured intracranial aneurysms; ROC, Receiver Operating Characteristic; AUC, area under the ROC curve; SE, standard error; Cut-off, optimal cut-off based on the highest Youden Index; YI, Youden Index; SE, sensitivity; SP, specificity; AC, diagnostic accuracy; PPV, positive predictive value; NPV, negative predictive value.

serum S100 and NSE concentrations $(0.07 \mu \mathrm{g} / \mathrm{l}$ and 13.37 $\mathrm{ng} / \mathrm{ml}$, respectively) compared to the control group (0.09 $\mu \mathrm{g} / \mathrm{l}$ and $16.59 \mathrm{ng} / \mathrm{ml}$, respectively) (Figure $3 \mathrm{~A}$ and $\mathrm{B}$ ). According to the manufacturer's instructions, expected values in healthy subjects with undamaged BBB should not exceed $0.105 \mu \mathrm{g} / \mathrm{l}$ for $\mathrm{S} 100$ and $16.3 \mathrm{ng} / \mathrm{ml}(95 \% \mathrm{CI}$ : $15.7-17.0 \mathrm{ng} / \mathrm{ml}$ ) for NSE. The correlation coefficient analysis showed that in the UIA group neither S100 nor NSE displayed a relationship with serum IL-6, CSF IL-6, or IL-6 Quotient ( $>0.05$ ).

\section{CSF IL-6 is Synthesized Intrathecally in UIA Patients. IL-8 Quotient is Useful to Distinguish UIA Patients from Individuals Without Vascular Lesions in the Brain}

To detect whether IL-6 is synthesized locally within the central nervous system in UIA patients, we evaluated its levels in the cerebrospinal fluid and found that CSF IL-6 median concentration was significantly higher $(4.55 \mathrm{pg} / \mathrm{ml})$ compared to serum concentration ( $2.39 \mathrm{pg} / \mathrm{ml}$, Figure 4).
Table 3 Multivariate Logistic Regression Analysis Results for UIA Diagnosis

\begin{tabular}{|c|c|c|c|}
\hline Variable & OR & $95 \% \mathrm{Cl}$ & p-value \\
\hline \multicolumn{4}{|c|}{ Multivariate Logistic Regression Analysis } \\
\hline I. IL-6 Quotient & $476,446.7$ & $1.84-1.23 \mathrm{e}+11$ & 0.040 \\
\hline 2. CSF SIO0 $[\mu \mathrm{g} / \mathrm{l}]$ & 0.099 & $0.02-0.49$ & 0.005 \\
\hline 3. SI00 Quotient & 1.094 & $1.02-1.17$ & 0.007 \\
\hline $\begin{array}{l}\text { 4. Interaction (Age* IL-6 } \\
\text { Quotient) }\end{array}$ & 0.835 & $0.69-0.99$ & 0.048 \\
\hline
\end{tabular}

Notes: *Interaction; the bold font of $p$-values mean that it is statistically significant $(p<0.05)$.

Abbreviations: OR, odds ratio; $\mathrm{Cl}$, confidence interval.

We also found that median IL-6 Quotient was significantly higher (1.78) in UIA patients compared to the control individuals (0.87) (Figure 5).

To assess the diagnostic utility of IL-6 Quotient evaluation in differentiating UIA patients from individuals without vascular lesions in the brain, we performed receiver operating characteristic (ROC) curve analysis and assessed the area under the ROC curve (AUC) (Figure 6).

Table 4 The Interpretation of the Interaction (Age* IL-6 Quotient) in Multiple Logistic Regression Analysis

\begin{tabular}{|l|c|l|}
\hline $\begin{array}{l}\text { Age } \\
\text { [years] }\end{array}$ & OR & Interpretation \\
\hline 20 & $12,805.76$ & $\begin{array}{l}\text { For people aged 20, with an increase in IL-6 } \\
\text { Quotient by I, the chance of UIA diagnosis is } \\
12,806 \text { times greater }\end{array}$ \\
\hline 30 & 2099.43 & $\begin{array}{l}\text { For people aged 30, with an increase in IL-6 } \\
\text { Quotient by I, the chance of UIA diagnosis is } \\
2099 \text { times greater }\end{array}$ \\
\hline 40 & 344.19 & $\begin{array}{l}\text { For people aged 40, with an increase in IL-6 } \\
\text { Quotient by I, the chance of UIA diagnosis is } \\
344 \text { times greater }\end{array}$ \\
\hline 50 & 56.43 & $\begin{array}{l}\text { For people aged 50, with an increase in IL-6 } \\
\text { Quotient by I, the chance of UIA diagnosis is } \\
56 \text { times greater }\end{array}$ \\
\hline 70 & 9.25 & $\begin{array}{l}\text { For people aged 60, with an increase in IL-6 } \\
\text { Quotient by I, the chance of UIA diagnosis is } \\
9.3 \text { times greater }\end{array}$ \\
\hline
\end{tabular}

Note: *Interaction. 


\section{IL-6 Quotient Growth is Predictive for UIA Diagnosis}

Logistic regression analysis was performed to assess whether IL-6 Quotient growth could be predictive for UIA diagnosis. Due to the correlation coefficient analysis conducted in our study group presenting that the older the UIA patient, the lower the IL-6 Quotient ( $\mathrm{R}=-0.32, \mathrm{p}=0.0121)$, we deduced that there is some correlation between age and IL-6 Quotient levels. Thus, the multivariate logistic regression analysis model was adjusted for age. In the created model, predictor variables influencing UIA diagnosis included the values of IL-6 Quotient, CSF S100, S100 Quotient, and age. Multivariate logistic regression analysis did not show weight, height, body mass index (BMI), obesity, alcohol, smoking, blood pressure, diabetes, head trauma, or cardiovascular diseases as predictive for UIA diagnosis.

We showed that: 1) if IL-6 Quotient increases by 1 then the chance of UIA diagnosis will change depending on the patient's age, and it will increase $e^{\beta_{1}+\beta_{4} a g e}$ times $(\beta 1=13.07, \beta 4=-0.18) ; 2)$ if CSF S100 concentration increases by $1 \mu \mathrm{g} / 1$ then the chance of UIA diagnosis decreases by 10.1 times; 3) if CSF S100 Quotient increases by 1 then the chance of UIA diagnosis increases by 1.095 times (increases by $9.5 \%$ ) (Table 3). Table 4 presents the interpretation of the interactions (Age* IL-6 Quotient) in multiple logistic regression analysis (Table 4).

\section{CSF IL-6 Concentration Reflects UIA} Formation but is Not Associated with Brain Aneurysm Progression

Correlation coefficient analysis showed that CSF IL-6 concentration positively correlated with the number of aneurysms $(\mathrm{R}=0.29, \mathrm{p}=0.0217)$ and negatively correlated with the aneurysm size $(\mathrm{R}=-0.48, \mathrm{p}=0.0005)$. IL-6 Quotient revealed a negative correlation with aneurysm size $(\mathrm{R}=-0.43, \mathrm{p}=0.0021)$. Patients with multiple brain aneurysms had significantly higher CSF IL-6 levels compared to individuals with a single aneurysm. Both CSF IL6 concentration and IL-6 Quotient were significantly lower in patients with aneurysm size $\geq$ median value $(5.3 \mathrm{~mm})$ compared to those with a smaller aneurysm size (Table 5).

\section{Discussion}

Our study was the first which showed that UIA patients had a significantly higher IL-6 Quotient (CSF IL-6 concentration divided by serum IL-6 concentration) compared to patients without vascular lesions in the brain. A further detailed analysis conducted in our study showed that IL-6 Quotient seems to be clinically useful for indicate patients with UIA.

Interestingly, in UIA patients we observed an almost 2-fold higher CSF IL-6 concentration compared to serum levels. On this basis, we conclude that a high concentration of IL-6 in CSF may suggest its local synthesis within the central nervous system (CNS), and thus its potential participation in the formation of intracranial aneurysms. Moreover, patients with multiple brain aneurysms had significantly higher CSF IL-6 levels compared to individuals with a single aneurysm, which adds weight to the contention that IL-6 concentration may reflect UIA formation.

The source of IL-6 concentration within the CNS in patients with an unruptured brain aneurysm may be vascular endothelial cells themselves, migrating along with inflammatory cells to the site of the aneurysm formation:

Table 5 CSF IL-6 Concentration and IL-6 Quotient Depending on Aneurysm Size and the Number of Aneurysms

\begin{tabular}{|l|c|c|c|}
\hline \multirow{2}{*}{} & Single & Number of Aneurysms & \multirow{2}{*}{ P-value } \\
\cline { 2 - 4 } & $4.14(3.25-4.96)$ & $5.08(4.20-6.70)$ & 0.0227 \\
\hline CSF IL-6 [pg/ml] & 1.7 I (0.93-2.8I) & $1.87(1.27-2.63)$ & 0.3394 \\
\hline IL-6 Quotient & \multicolumn{2}{|c|}{ Aneurysm Size [mm] } & \\
\hline \multicolumn{2}{|c|}{$<.3$} & $4.00(3.05-4.78)$ & 0.0022 \\
\hline CSF IL-6 [pg/ml] & $5.19(4.44-7.3 I)$ & $1.33(0.86-2.08)$ & 0.0183 \\
\hline IL-6 Quotient & $2.5 I(1.54-3.5 I)$ & & \\
\hline
\end{tabular}

Note: The bold font of $p$-value means that it is statistically significant $(p<0.05)$. 
monocytes/macrophages and lymphocytes, which are able to synthesize IL-6 in response to a stimulus. ${ }^{2,14,15}$ Additionally, astrocytes stimulated by various cytokines such as IL- $1 \beta$ or TNF- $\alpha$, produce IL- $6 .^{11,14,28}$ An additional source of IL- 6 is activated microglia releasing IL6 in response to changes in the CNS. ${ }^{11}$ The current study showed the high local synthesis of IL- 6 within the CNS in UIA patients. Taking into account the role of IL-6 in driving inflammation, ${ }^{29}$ we may hypothesize its role in intracranial aneurysm formation. In this regard, IL-6 may be essential, as it enhances the inflammatory process within the vascular endothelium and the synthesis of molecules involved in the formation of brain aneurysms $^{21,30-34}$ (Figure 7).

Since the disturbances in the blood-brain barrier (BBB) and the blood-cerebrospinal fluid barrier (BCSFB) may affect final protein concentration in the $\mathrm{CSF}^{25}$ we also analyzed IL-6 levels depending on the condition of the above-mentioned barriers. Particularly, that the in vitro studies by Tada et al showed decreased expression of BBB tight junction proteins, ie, occludins and $\mathrm{ZO}-1$, which in their opinion may be crucial in the formation of aneurysms in

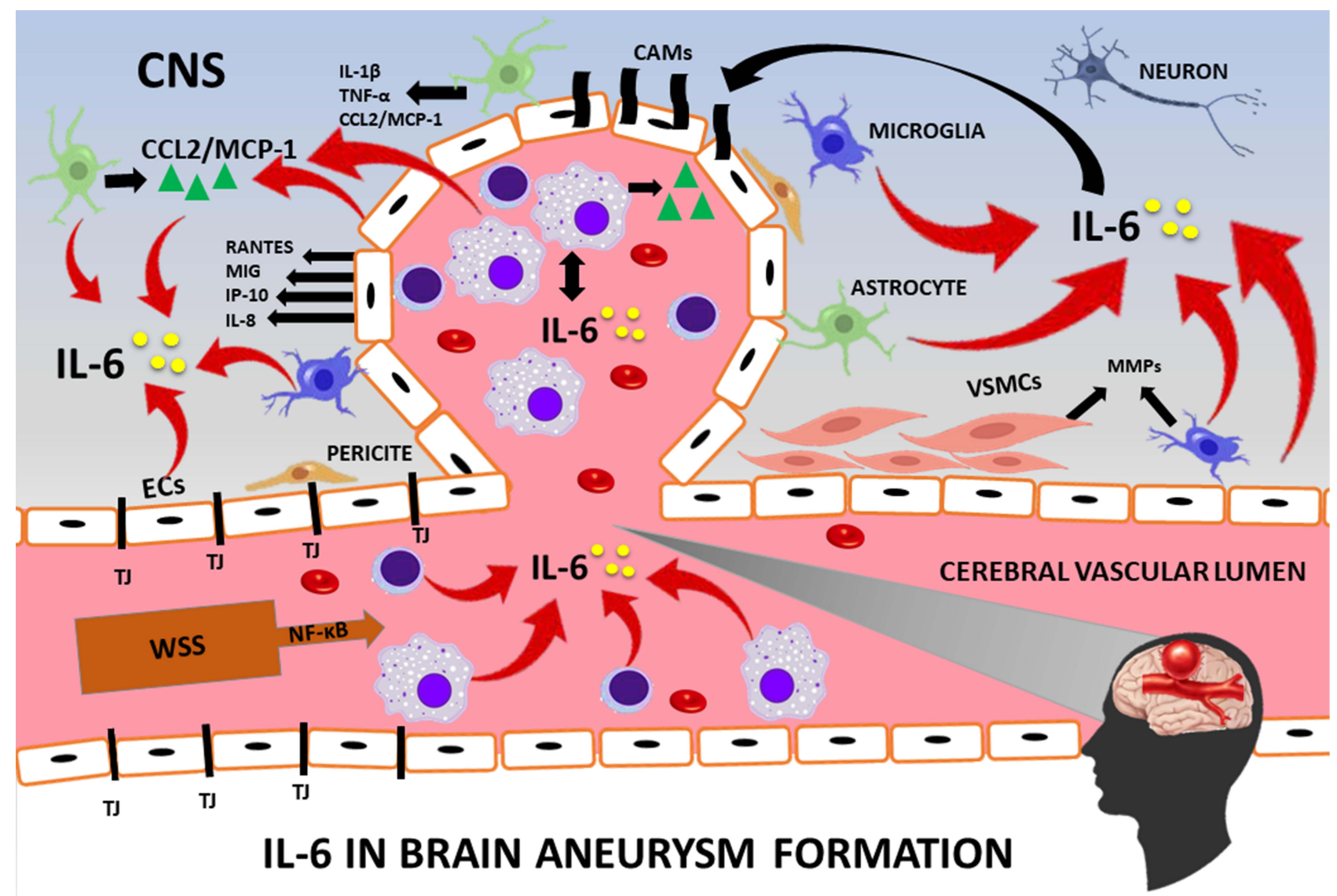

Figure 7 Suggested contribution of interleukin 6 (IL-6) to brain aneurysm formation.

Notes: The formation of a brain aneurysm begins when wall shear stress (WSS) initiates the production of NF- $\kappa B$, being a transcription factor that initiates the process of inflammation. The sources of interleukin 6 (IL-6) in the central nervous system (CNS) are I) vascular endothelial cells (ECs), 2) leukocytes: monocytes/macrophages and lymphocytes migrating to the site of the aneurysm formation in response to high WSS, 3 ) astrocytes stimulated by various cytokines (eg, IL-I $\beta$, TNF- $\alpha$ ), and 4 ) microglia in response to changes in the CNS. IL-6, in turn, enhances the inflammatory dysfunction of the endothelium via the production of adhesion molecules by the ECs (eg, VCAMI, VCAM2), cytokines (eg, IL-8), chemokines (eg, RANTES, MIG, IP-I0), and other molecules involved in the formation of a brain aneurysm. IL-6 also stimulates macrophages to secrete CCL-2/MCP-I established as a critical factor responsible for the formation of IA. IL-6 also increases the c-myc mRNA expression directly stimulating DNA synthesis, which promotes vascular smooth muscle cell (VSMCs) proliferation. Additional VSMCs phenotypic modulation results in the loss of their contraction capacity via the downregulation in expression of contractile genes (eg, myocardin), and upregulation of genes that may affect the rigidity or elasticity of the vascular wall (eg, collagen alfa2(I) gene) as well as proinflammatory genes (eg, MMPs, CCL2/MCP-I, VCAM I, and ILs). ${ }^{2-4,8, I 1,14,21,26,28-30}$

Abbreviations: CAMs, cell adhesion molecules; CCL2/MCP-I; (C-C motif) ligand 2/monocyte chemoattractant protein-I; CNS, central nervous system; ECs, endothelial cells; IL-I $\beta$, interleukin I beta; IL-8, interleukin 8; ILs, interleukins; IP-I0, interferon gamma-induced protein I0; MIG, monokine induced by gamma interferon; MMPs, matrix metalloproteinases; mRNA, messenger ribonucleic acid; NF- $\kappa B$, nuclear factor kappa-light-chain-enhancer of activated leukocytes, normal T-cell expressed and secreted; RANTES, regulated on activation; TNF- $\alpha$, tumor necrosis factor- $\alpha$; VCAMI, vascular cell adhesion molecule I; VCAM2, vascular cell adhesion molecule 2; VSMCs, vascular smooth muscle cells; WSS, wall shear stress. 
rats. ${ }^{35}$ In turn, Saitou et al and Schulzke et al demonstrated normal tight junction morphology and normal barrier transport in mice with congenital total occludin deficiency, ${ }^{36,37}$ which contradicts the theory of Tada et al. ${ }^{35}$

A detailed analysis of markers used to assess both barriers: QAlb, S100, and NSE proteins indicated that UIA patients included in our study possessed barriers in the proper condition. Moreover, in UIA patients neither QAlb nor S100 and NSE concentration correlated with CSF IL-6 levels.

The obtained results strengthen our hypothesis that the increased concentration of CSF IL-6 compared to serum IL-6 in patients with unruptured brain aneurysms, resulted from its local synthesis in the CNS, suggesting the role of this cytokine in the formation of an intracranial aneurysm. Additionally, our results suggest that the evaluation of serum S100 and NSE proteins does not apply to patients with UIA, unlike patients with an aneurysm rupture and aneurysm SAH. ${ }^{38-40}$

Chalouhi et al observed a significantly lower concentration of IL-6 in the blood collected from the lumen of the brain aneurysm compared to the blood collected from the normal femoral vessel, but their group included both patients with non-ruptured $(\mathrm{n}=13)$ and ruptured aneurysms $(n=3) .{ }^{31}$ However, when the authors analyzed the concentration of cytokine only in those patients with UIA, they did not observe significant differences for IL-6. ${ }^{31} \mathrm{We}$ conducted our study among a homogeneous, relatively large group $(n=67)$ including only unruptured brain aneurysm patients, in both CSF and serum, which is an undoubted advantage. Our research suggests that the assessment of IL-6 concentration only in serum of patients with an unruptured brain aneurysm is not clinically applicable, because it does not reflect the local IL-6 synthesis within the CSN. Especially since we have shown that UIA patients have both BCSFB and BBB in the proper condition.

\section{Study Limitation}

A limitation of our study is the "control group", which included people suffering from persistent facial pain caused by irritation of the trigeminal nerve. In our study, CSF was secured during neurosurgery; its collection by lumbar puncture is not routinely performed in UIA patients and would not be approved by the local bioethics committee. We wanted to collect CSF from the control group in the same way as carried out with UIA patients, which resulted in selecting patients with trigeminal neuralgia as the comparison group.
The second study limitation is a lack of correlation between IL-6 levels and results of IL-6 polymorphism analysis, taking into consideration that studies on the polymorphism of the IL-6 gene suggest that this molecule seems to be a candidate gene contributing to the pathogenesis of IA. We have designed such a study, and we are currently in the process of sample collection and applying for funds.

\section{Strengths of the Study}

The strength of our study lies in the assessment of IL-6 concentration in both CSF and serum, and the calculation of the IL-6 Quotient. It should be noted that CSF samples contaminated with blood were excluded from further analysis. Our study showed that the calculation of the IL-6 Quotient seems to be clinically useful for indicating individuals with UIA. We also conducted our study among a homogeneous, relatively large group $(n=67)$ including only unruptured brain aneurysm patients, which is an undoubted advantage.

\section{Conclusion}

Our study was the first which showed that the calculation of IL-6 Quotient seems to be clinically useful for indicating individuals with UIA. In both the study and control group the blood-brain barrier was intact, thus the CSFblood gradient of the IL- 6 concentration in UIA patients was likely to be the expression of local synthesis of the cytokine within the central nervous system. We hypothesize IL-6 participation in intracranial aneurysm formation via its multifactorial effect supporting the inflammation within the aneurysmal wall, which relays on actions on leukocytes, vascular endothelial cells, smooth muscle cells, and CNS cells (Figure 7). This hypothesis may be further confirmed by the fact that patients with multiple brain aneurysms had significantly higher CSF IL-6 levels compared to individuals with a single aneurysm.

\section{Data Sharing Statement}

The datasets generated and/or analyzed during the current study are not publicly available, but are available from the corresponding author (J.Ka.) on a request.

\section{Ethics Approval and Consent to Participate}

The study was conducted according to the guidelines of the Declaration of Helsinki and the protocol was approved by the Bioethics Human Research Committee of the 
Medical University of Bialystok (Permission No. R-I-002/ 383/2015). All subjects gave their informed consent for inclusion before they participated in the study.

\section{Acknowledgments}

We are grateful to Martin Lenkiewicz and Jan Dobrodumow for their language assistance.

\section{Funding}

This research received no external funding. This work was supported by the Medical University of Bialystok (statutory work: SUB/1/DN/21/001/2209).

\section{Disclosure}

PL received consultation and/or lecture honoraria from IBL International, Fujirebio Europe, AJ Roboscreen, Biogen, and Roche. The authors report no other conflicts of interest in this work.

\section{References}

1. Steiner T, Juvela S, Unterberg A, Jung C, Forsting M, Rinkel G. European stroke organization guidelines for the management of intracranial aneurysms and subarachnoid haemorrhage. Cerebrovasc Dis. 2013;35(2):93-112. doi:10.1159/000346087

2. Signorelli F, Turjman F, Gory B, Labeyrie PE, Pelissou-Guyotat I, Riva R. Hemodynamics, inflammation, vascular remodeling, and the development and rupture of intracranial aneurysms: a review. Neuroimmunol Neuroinflamm. 2015;2(2):59. doi:10.4103/23478659.154885

3. Hashimoto $\mathrm{T}$, Meng $\mathrm{H}$, Young WL. Intracranial aneurysms: links among inflammation, hemodynamics and vascular remodeling. Neurol Res. 2006;28(4):372-380. doi:10.1179/016164106X14973

4. Chalouhi N, Ali MS, Jabbour PM, et al. Biology of intracranial aneurysms: role of inflammation. J Cereb Blood Flow Metab. 2012;32(9):1659-1676. doi:10.1038/jcbfm.2012.84

5. Chalouhi N, Ali MS, Starke RM, et al. Cigarette smoke and inflammation: role in cerebral aneurysm formation and rupture. Mediators Inflamm. 2012;2012:1-12. doi:10.1155/2012/271582

6. Zhang HF, Zhao MG, Liang GB, Song ZQ, Li ZQ. Expression of pro-inflammatory cytokines and the risk of intracranial aneurysm. Inflammation. 2013;36(6):1195-1200. doi:10.1007/s10753-0139655-6

7. Sawyer DM, Amenta PS, Medel R, Dumont AS. Inflammatory mediators in vascular disease: identifying promising targets for intracranial aneurysm research. Mediators Inflamm. 2015;2015:1-10. doi:10.1155/2015/896283

8. Signorelli F, Sela S, Gesualdo L, et al. Hemodynamic stress, inflammation, and intracranial aneurysm development and rupture: a systematic review. World Neurosurg. 2018;115:234-244. doi:10.1016/j.wneu.2018.04.143

9. Labeyrie P-E, Goulay R, Martinez de Lizarrondo S, et al. Vascular tissue-type plasminogen activator promotes intracranial aneurysm formation. Stroke. 2017;48(9):2574-2582. doi:10.1161/ STROKEAHA.117.017305

10. Signorelli F, Pailler-Mattei C, Gory B, et al. Biomechanical characterization of intracranial aneurysm wall: a multiscale study. World Neurosurg. 2018;119:e882-e889. doi:10.1016/j.wneu.2018.07.290
11. Hendryk S, Jarzab B, Josko J. Increase of the IL-1 beta and IL-6 levels in CSF in patients with vasospasm following aneurysmal SAH. Neuro Endocrinol Lett. 2004;25(1-2):141-147.

12. Chyatte D, Bruno G, Desai S, Todor DR. Inflammation and intracranial aneurysms. Neurosurgery. 1999;45(5):1137-1147. doi:10.1097/ 00006123-199911000-00024

13. Aoki T, Nishimura M. Targeting chronic inflammation in cerebral aneurysms: focusing on NF-kappaB as a putative target of medical therapy. Expert Opin Ther Targets. 2010;14(3):265-273. doi:10.1517/ 14728221003586836

14. Tanaka T, Narazaki M, Kishimoto T. IL-6 in inflammation, immunity, and disease. Cold Spring Harb Perspect Biol. 2014;6(10):a016295. doi:10.1101/cshperspect.a016295

15. Akira S, Taga T, Kishimoto T. Interleukin-6 in biology and medicine. Adv Immunol. 1993;54:1-78. doi:10.1016/s0065-2776(08)60532-5

16. Osuka K, Suzuki Y, Tanazawa T, et al. Interleukin-6 and development of vasospasm after subarachnoid haemorrhage. Acta Neurochir. 1998;140(9):943-951. doi:10.1007/s007010050197

17. Fassbender K, Hodapp B, Rossol S, et al. Inflammatory cytokines in subarachnoid haemorrhage: association with abnormal blood flow velocities in basal cerebral arteries. J Neurol Neurosurg Psychiatry. 2001;70(4):534-537. doi:10.1136/jnnp.70.4.534

18. Duriš K, Neuman E, Vybíhal V, et al. Early dynamics of interleukin-6 in cerebrospinal fluid after aneurysmal Subarachnoid hemorrhage. J Neurol Surg Part a Cent Eur Neurosurg. 2018;79(2):145-151. doi:10.1055/s-0037-1604084

19. Ni W, Gu YX, Song DL, Leng B, Li PL, Mao Y. The relationship between IL-6 in CSF and occurrence of vasospasm after subarachnoid hemorrhage. In: Early Brain Injury or Cerebral Vasospasm. Vienna: Springer;2011:203-208. doi:10.1007/978-3-7091-0353-1_35

20. Kao HW, Lee KW, Kuo CL, et al. Interleukin-6 as a prognostic biomarker in ruptured intracranial aneurysms. PLoS One. 2015;10 (7):6-13. doi:10.1371/journal.pone.0132115

21. Kamińska J, Lyson T, Chrzanowski R, et al. Ratio of IL-8 in CSF versus serum is elevated in patients with unruptured brain aneurysm. J Clin Med. 2020;9(6):1761. doi:10.3390/jcm9061761

22. Murayama Y, Takao H, Ishibashi T, et al. Risk analysis of unruptured intracranial aneurysms: prospective 10-year cohort study. Stroke. 2016;47(2):365-371. doi:10.1161/STROKEAHA. 115.010698

23. Reiber H. Knowledge-base for interpretation of cerebrospinal fluid data patterns. Essentials in neurology and psychiatry. Arq Neuropsiquiatr. 2016;74(6):501-512. doi:10.1590/0004-282×20160066

24. Kamińska J, Koper OM, Piechal K, Kemona H. Multiple sclerosis etiology and diagnostic potential. Postepy Hig Med Dosw. 2017;71 (1):551-563. doi:10.5604/01.3001.0010.3836

25. Reiber H, Peter JB. Cerebrospinal fluid analysis: disease-related data patterns and evaluation programs. $J$ Neurol Sci. 2001;184 (2):101-122. doi:10.1016/S0022-510X(00)00501-3

26. Lindblad C, Nelson DW, Zeiler FA, et al. Influence of blood-brain barrier integrity on brain protein biomarker clearance in severe traumatic brain injury: a longitudinal prospective study. J Neurotrauma. 2020;37(12):1381-1391. doi:10.1089/neu.2019.6741

27. Dimopoulou I, Korfias S, Dafni U, et al. Protein S-100b serum levels in trauma-induced brain death. Neurology. 2003;60(6):947-951. doi:10.1212/01.WNL.0000049931.77887.7F

28. Aloisi F, Carè A, Borsellino G, et al. Production of hemolymphopoietic cytokines normal human astrocytes in response to IL-1 beta and tumor necrosis factor-alpha. J Immunol. 1992;149(7):2358-2366.

29. Romano M, Sironi M, Toniatti C, et al. Role of IL-6 and its soluble receptor in induction of chemokines and leukocyte recruitment. Immunity. 1997;6(3):315-325. doi:10.1016/S1074-7613(00)80334-9

30. Biswas P, Delfanti F, Bernasconi S, et al. Interleukin-6 induces monocyte chemotactic protein-1 in peripheral blood mononuclear cells and in the U937 cell line. Blood. 1998;91(1):258-265. doi:10.1182/blood.V91.1.258 
31. Chalouhi N, Points L, Pierce GL, Ballas Z, Jabbour P, Hasan D. Localized increase of chemokines in the lumen of human cerebral aneurysms. Stroke. 2013;44(9):2594-2597. doi:10.1161/ STROKEAHA.113.002361

32. Morgan L, Cooper J, Montgomery H, Kitchen N, Humphries SE. The interleukin- 6 gene $-174 \mathrm{G}>\mathrm{C}$ and $-572 \mathrm{G}>\mathrm{C}$ promoter polymorphisms are related to cerebral aneurysms. J Neurol Neurosurg Psychiatry. 2006;77(8):915-917. doi:10.1136/jnnp.2005.081976

33. Rattazzi M, Puato M, Faggin E, Bertipaglia B, Zambon A, Pauletto P. C-reactive protein and interleukin-6 in vascular disease. J Hypertens. 2003;21(10):1787-1803. doi:10.1097/00004872-200310000-00002

34. Tylicka M, Matuszczak E, Kamińska J, et al. Intraoperative peritoneal interleukin-6 concentration changes in relation to the high-mobility group protein B1 and heat shock protein 70 levels in children undergoing cholecystectomy. Mediators Inflamm. 2020;2020:1-9. doi:10.1155/2020/9613105

35. Tada Y, Yagi K, Kitazato KT, et al. Reduction of endothelial tight junction proteins is related to cerebral aneurysm formation in rats. J Hypertens. 2010;28(9):1883-1891. doi:10.1097/HJH;0b013e32833c2273
36. Saitou M, Furuse M, Sasaki H, et al. Complex phenotype of mice lacking occludin, a component of tight junction strands. Mol Biol Cell. 2000;11(12):4131-4142. doi:10.1091/mbc.11.12.4131

37. Schulzke JD, Gitter AH, Mankertz J, et al. Epithelial transport and barrier function in occludin-deficient mice. Biochim Biophys Acta Biomembr. 2005;1669(1):34-42. doi:10.1016/j.bbamem.2005.01.008

38. Yardan T, Erenler AK, Baydin A, Aydin K, Cokluk C. Usefulness of S100B protein in neurological disorders. J Pak Med Assoc. 2011;61 (3):276-281.

39. Rezaei O, Pakdaman H, Gharehgozli K, et al. S100 B: a new concept in neurocritical care. Iran J Neurol. 2017;16(2):83-89.

40. Weiss N, Sanchez-Peña $P$, Roche $S$, et al. Prognosis value of plasma S100B protein levels after subarachnoid aneurysmal hemorrhage. Anesthesiology. 2006;104(4):658-666. doi:10.1097/00000542200604000-00008
Journal of Inflammation Research

\section{Publish your work in this journal}

The Journal of Inflammation Research is an international, peerreviewed open-access journal that welcomes laboratory and clinical findings on the molecular basis, cell biology and pharmacology of inflammation including original research, reviews, symposium reports, hypothesis formation and commentaries on: acute/chronic inflammation; mediators of inflammation; cellular processes; molecular
Dovepress

mechanisms; pharmacology and novel anti-inflammatory drugs; clinical conditions involving inflammation. The manuscript managemen system is completely online and includes a very quick and fair peerreview system. Visit http://www.dovepress.com/testimonials.php to read real quotes from published authors. 